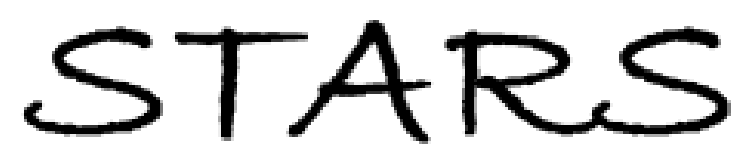

University of Central Florida

STARS

Faculty Bibliography 2010s

Faculty Bibliography

$1-1-2010$

\title{
Epitaxial growth of a silicene sheet
}

Boubekeur Lalmi

Hamid Oughaddou

Hanna Enriquez

Abdelkader Kara

University of Central Florida

Sébastien Vizzini

See next page for additional authors

Find similar works at: https://stars.library.ucf.edu/facultybib2010

University of Central Florida Libraries http://library.ucf.edu

This Article is brought to you for free and open access by the Faculty Bibliography at STARS. It has been accepted for inclusion in Faculty Bibliography 2010 s by an authorized administrator of STARS. For more information, please contactSTARS@ucf.edu.

\section{Recommended Citation}

Lalmi, Boubekeur; Oughaddou, Hamid; Enriquez, Hanna; Kara, Abdelkader; Vizzini, Sébastien; Ealet, Bénidicte; and Aufray, Bernard, "Epitaxial growth of a silicene sheet" (2010). Faculty Bibliography 2010s. 402.

https://stars.library.ucf.edu/facultybib2010/402

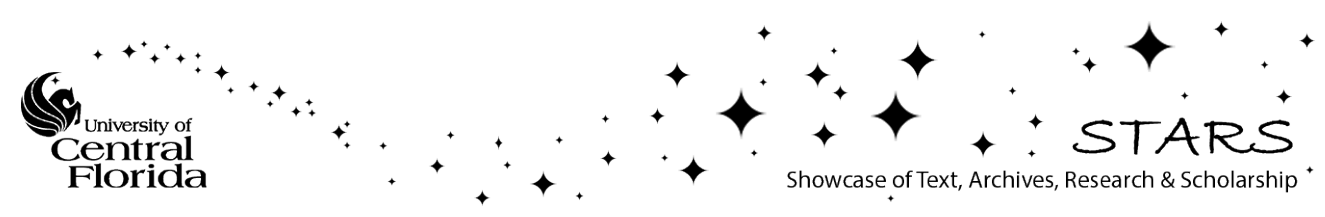




\section{Authors}

Boubekeur Lalmi, Hamid Oughaddou, Hanna Enriquez, Abdelkader Kara, Sébastien Vizzini, Bénidicte Ealet, and Bernard Aufray 


\section{Epitaxial growth of a silicene sheet}

Cite as: Appl. Phys. Lett. 97, 223109 (2010); https://doi.org/10.1063/1.3524215

Submitted: 19 October 2010 . Accepted: 11 November 2010 . Published Online: 02 December 2010

Boubekeur Lalmi, Hamid Oughaddou, Hanna Enriquez, Abdelkader Kara, Sébastien Vizzini, Bénidicte Ealet, and Bernard Aufray

\section{ARTICLES YOU MAY BE INTERESTED IN}

Evidence of graphene-like electronic signature in silicene nanoribbons

Applied Physics Letters 96, 261905 (2010); https://doi.org/10.1063/1.3459143

$s p^{2}$-like hybridization of silicon valence orbitals in silicene nanoribbons

Applied Physics Letters 98, 081909 (2011); https://doi.org/10.1063/1.3557073

Electronic properties of hydrogenated silicene and germanene

Applied Physics Letters 98, 223107 (2011); https://doi.org/10.1063/1.3595682

\section{Applied Physics Reviews} Now accepting original research 


\title{
Epitaxial growth of a silicene sheet
}

\author{
Boubekeur Lalmi, ${ }^{1}$ Hamid Oughaddou, ${ }^{2,3, a)}$ Hanna Enriquez, ${ }^{2,4}$ Abdelkader Kara, ${ }^{5,3}$ \\ Sébastien Vizzini, ${ }^{6}$ Bénidicte Ealet, ${ }^{1}$ and Bernard Aufray ${ }^{1, a)}$ \\ ${ }^{1}$ CINaM-CNRS, Campus de Luminy, Case 913, Marseille 13288, France \\ ${ }^{2}$ CEA, DSM-IRAMIS-SPCSI, Bât. 462, Saclay, Gif-sur-Yvette 91191, France \\ ${ }^{3}$ Département de Physique, Université de Cergy-Pontoise, Cergy-Pontoise 95000, France \\ ${ }^{4}$ Département de Physique, Université Paris-Sud, Orsay Cedex 91405, France \\ ${ }^{5}$ Department of Physics, University of Central Florida, Orlando, Florida 32816, USA \\ ${ }^{6}$ IM2NP, Faculté des Sciences de Saint-Jérôme, Aix-Marseille Université, Case 142, Marseille 13397, \\ France
}

(Received 19 October 2010; accepted 11 November 2010; published online 2 December 2010)

\begin{abstract}
Using atomic resolved scanning tunneling microscopy, we present here the experimental evidence of a silicene sheet (graphenelike structure) epitaxially grown on a close-packed silver surface $[\mathrm{Ag}(111)]$. This has been achieved via direct condensation of a silicon atomic flux onto the single-crystal substrate in ultrahigh vacuum conditions. A highly ordered silicon structure, arranged within a honeycomb lattice, is synthesized and present two silicon sublattices occupying positions at different heights $(0.02 \mathrm{~nm})$ indicating possible $\mathrm{sp}^{2}-\mathrm{sp}^{3}$ hybridizations. (C) 2010 American Institute of Physics. [doi:10.1063/1.3524215]
\end{abstract}

Silicene presents a honeycomb structure similar to that of graphene, which has demonstrated applications in nanotechnology and is currently the most investigated material in physics and nanoscience. ${ }^{1}$ The synthesis of this silicon-based crystalline material opens the way for studies of its physical/ chemical properties, some of which have been predicted from theory to be similar to those of grapheme, that have potential applications in nanotechnology with the added advantage of being compatible with existing semiconductor devices.

Silicene has recently attracted strong theoretical attention. $^{2-4}$ For example, Cahangirov et al., ${ }^{4}$ using density functional theory, have shown that a silicon quasi-twodimensional structure is stable (without of any imaginary vibrational frequency) only if a low buckling (LB), 0.044 $\mathrm{nm}$, is allowed. They also report that for this LB structure the $\mathrm{Si}-\mathrm{Si}$ nearest-neighbor distance is reduced to $0.225 \mathrm{~nm}$ from that of the bulk and that the electronic density of states indicates the system is ambipolar. Their calculated band structures exhibit a crossing at $K$ and $K^{\prime}$ points (reflecting the semimetallic character of silicene) and a linear dependency near the crossing (reflecting a massless Dirac fermion character). The same authors find that silicene nanoribbons (NRs) exhibit electronic and magnetic properties likewise very similar to those of graphene. From an experimental point of view, however, silicene sheets have not been synthesized until now, although single-wall ${ }^{5}$ and multiwall ${ }^{6}$ silicon nanotubes have been produced and monolayers of silicon have been synthesized by exfoliation ${ }^{7}$ (though no two dimensional character has been detected).

Our group began studying silicon adsorption on silver surfaces several years ago. On $\operatorname{Ag}(001)$ we observed superstructures that we eventually came to characterize as deformed hexagonal structures. ${ }^{8}$ Subsequently, extensive investigations of $\mathrm{Si}$ submonolayer growth on $\mathrm{Ag}(110)$ revealed self-assembled NRs $1.6 \mathrm{~nm}$ in width and several hundred

\footnotetext{
a) Authors to whom correspondence should be addressed. Electronic addresses: hamid.oughaddou@u-cergy.fr and aufray@cinam.univ-mrs.fr.
}

nanometers in length. ${ }^{9-11}$ These NRs show strong resistance to oxidation on their edges, ${ }^{12}$ an intriguing chemical property given the strong propensity of silicon surfaces to oxidize. For these Si NRs, atomically resolved scanning tunneling microscopy (STM) images revealed a honeycomb structure ${ }^{11,13}$ that $a b$ initio calculations ${ }^{14}$ indicated to be composed of arched silicene NRs. Recently, using angle-resolved photoemission spectroscopy (ARPES), we have inferred that, as with that of graphene, the band dispersion along the direction of these NRs exhibits Dirac cones. ${ }^{15}$ The question thus arose as to whether a continuous film of such honeycomb structures, namely, silicene, could be achieved.

Very recently we have succeeded in growing a silicene sheet on a $\operatorname{Ag}(111)$ surface, in ultrahigh vacuum conditions, which we believe is the first evidence of silicene sheet synthesis. The apparatus in which the experiments have been performed is equipped with the standard tools for surface preparation and characterization: an ion gun for surface cleaning, low energy electron diffraction (LEED) instrument, an Auger electron spectrometer for chemical surface analysis, and STM for surface characterization at the atomic scale. The STM experiments were performed at room temperature with a commercial Omicron STM, Germany. The Ag(111) sample was cleaned by several cycles of sputtering $(600 \mathrm{eV}$ $\mathrm{Ar}^{+}$ions, $\mathrm{P} \sim 10^{-5}$ Torr) followed by annealing at $400{ }^{\circ} \mathrm{C}$ until a sharp $\mathrm{p}(1 \times 1)$ pattern was obtained. Silicon, evaporated by direct current heating of a piece of $\mathrm{Si}$ wafer, was deposited onto the $\operatorname{Ag}(111)$ surface held at $\sim 250{ }^{\circ} \mathrm{C}$. The silver substrate temperature was controlled by a thermocouple located close to the sample.

The experimental conditions for producing this silicon structure are quite stringent since the temperature of the substrate must be kept between 220 and $250{ }^{\circ} \mathrm{C}$ and the rate of deposition of silicon must be lower than 0.1 monolayer per minute. Finally, extreme care must be taken that exactly the amount of silicon required to produce a single monolayer is applied. Under these conditions, we produced the silicene sheets in three experiments. 


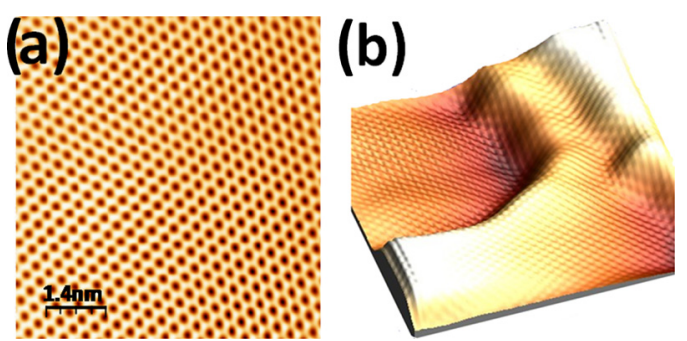

FIG. 1. (Color online) (a) Large-scale, filled-state STM image showing the graphenelike structure of one monolayer of silicon deposited on a closepacked silver surface, $\operatorname{Ag}(111)$. (b) 3D STM image showing $\operatorname{Ag}(111)$ steps covered by the silicene film $\left(11 \times 11 \mathrm{~nm}^{2}\right)$.

As displayed in Fig. 1(a), the atomically resolved STM image shows a highly ordered silicon monolayer, arranged within a honeycomb lattice that covers the entire scanned area of the substrate (several hundred $\mathrm{nm}^{2}$ ), while Fig. 1(b) presents an STM image showing that the silicene film covers the surface steps like graphene grown on metals. ${ }^{16,17} \mathrm{~A}$ detailed scrutiny of the STM images revealed that this structure is actually formed by two silicon sublattices occupying positions at different heights [Fig. 2(a)]. Analysis of several line-scan profiles, an example of which is shown in Fig. 2(b), yields a height difference of $0.02 \mathrm{~nm}$. This small observed corrugation (indicating possible $\mathrm{sp}^{2}-\mathrm{sp}^{3}$ hybridizations) is in line with the theoretically predicted one. ${ }^{4}$

These line-scan profiles contain several pieces of structural information which can be used to derive the $\mathrm{Si}-\mathrm{Si}$ nearest-neighbors distance in silicene. A peak-to-peak analysis provides a direct measure of this distance, which turns out to be between 0.19 and $0.2 \mathrm{~nm}$, while a valley-to-valley analysis indicates a $\mathrm{Si}-\mathrm{Si}$ distance of about $0.18-0.19 \mathrm{~nm}$. That this distance $(0.19 \pm 0.01 \mathrm{~nm})$ is about $17 \%$ shorter than that for Si bulk $(0.235 \mathrm{~nm})$ suggests that the $\mathrm{Ag}$ substrate may play a catalyst role in the formation and stabilization of the silicene sheet. That the LEED of the epitaxially grown film shows a $(2 \sqrt{3} \times 2 \sqrt{ } 3) \mathrm{R} 30^{\circ}$ superstructure confirms the existence of a long-range order. In addition the superstructure observed in the LEED pattern is not visible in the STM images probably due to a weak electronic coupling between the silicene sheet and the Ag substrate.

Combining atomic high-resolution STM images recorded on the same sample before and after the silicon deposition without any rotation [shown in Figs. 3(a) and 3(b), respectively] in conformity with the observed $(2 \sqrt{3}$ $\times 2 \sqrt{3}) \mathrm{R} 30^{\circ}$ LEED pattern, we propose a model of silicene sheet adsorbed on a $\operatorname{Ag}(111)$ surface [Fig. 3(c)]. Understanding the growth mechanisms and the stability of the silicene
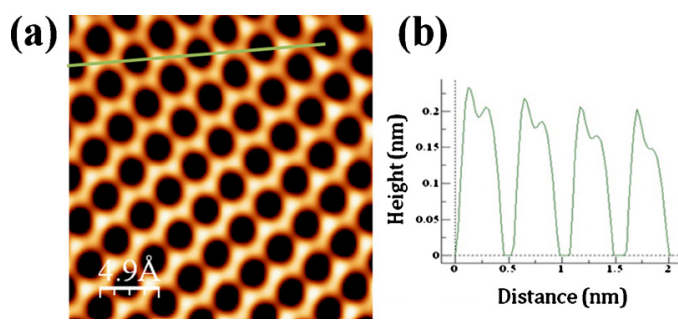

FIG. 2. (Color online) (a) Filled-state atomically resolved STM image showing honeycomb structure, revealing two sublattices. (b) Line-profile joining neighboring $\mathrm{Si}$ atoms along the direction indicated in (a).

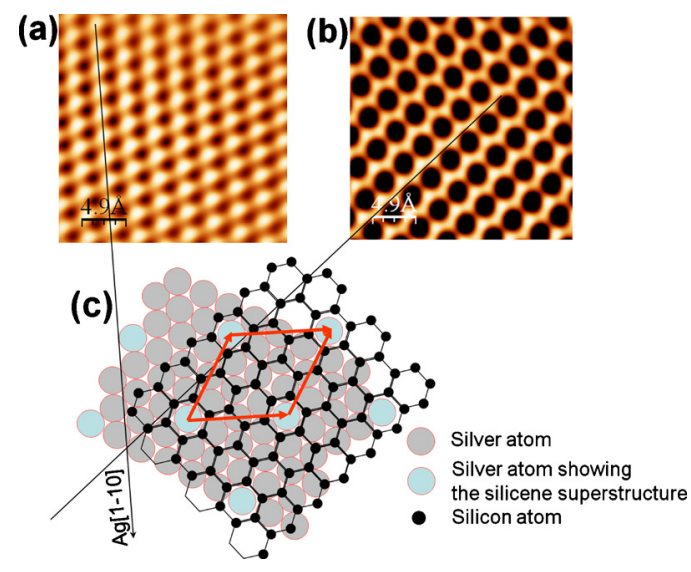

FIG. 3. (Color online) (a) Filled-state atomically resolved STM image of the clean $\operatorname{Ag}(111)$ surface. (b) Filled-state atomically resolved STM image of the same sample (without any rotation) after deposition of one silicon monolayer. (c) Proposed ball model of silicene on $\mathrm{Ag}(111)$ derived from both STM images (a) and (b) and from the observed $(2 \sqrt{3} \times 2 \sqrt{3})$ R $30^{\circ}$ LEED pattern.

sheet will allow us to assess the viability of silicene as a material with perspectives for potential applications in nanotechnology. Atomistic (ab initio and tight-binding) calculations are in progress to unravel the mechanisms by which such a structure is formed. The ARPES measurements are also being programmed in order to reveal the silicene band dispersion and to test the existence of Dirac cones.

We thank Lyman Baker for a critical reading of the manuscript. We thank also Guy Le Lay for fruitful discussions. A.K. thanks the University of Cergy-Pontoise for hospitality and support.

${ }^{1}$ A. K. Geim and K. S. Novoselov, Nature Mater. 6, 183 (2007).

${ }^{2}$ S. B. Fagan, R. J. Baierle, R. Mota, Z. J. R. da Silva, and A. Fazzio, Phys. Rev. B 61, 9994 (2000).

${ }^{3}$ S. Lebègue and O. Eriksson, Phys. Rev. B 79, 115409 (2009).

${ }^{4}$ S. Cahangirov, M. Topsakal, E. Aktürk, H. Şahin, and S. Ciraci, Phys. Rev. Lett. 102, 236804 (2009).

${ }^{5}$ M. De Crescenzi, P. Castrucci, M. Scarselli, M. Diociaiuti, Prajakta S. Chaudhari, C. Balasubramanian, T. M. Bhave, and S. V. Bhoraskar, Appl Phys. Lett. 86, 231901 (2005).

${ }^{6}$ S. Yamada and H. Fujiki, Jpn. J. Appl. Phys., Part 2 45, L837 (2006).

${ }^{7}$ H. Nakano, T. Mitsuoka, M. Harada, K. Horibuchi, H. Nozaki, N. Takahashi, T. Nonaka, Y. Seno, and H. Nakamura, Angew. Chem. 118, 6451 (2006)

${ }^{8}$ C. Léandri, H. Oughaddou, B. Aufray, J. M. Gay, G. Le Lay, A. Ranguis, and Y. Garreau, Surf. Sci. 601, 262 (2007).

${ }^{9}$ C. Léandri, G. Le Lay, B. Aufray, C. Girardeaux, J. Avila, M. E. Davila, M. C. Asensio, C. Ottaviani, and A. Cricenti, Surf. Sci. 574, L9 (2005).

${ }^{10}$ G. Le Lay, B. Aufray, C. Léandri, H. Oughaddou, J.-P. Bibérian, P. De Padova, M. E. Dávila, B. Ealet, and A. Kara, Appl. Surf. Sci. 256, 524 (2009).

${ }^{11}$ A. Kara, C. Léandri, M. E. Dávila, P. De Padova, B. Ealet, H. Oughaddou, B. Aufray, and G. Le Lay, J. Supercond. Novel Magn. 22, 259 (2009).

${ }^{12}$ P. De Padova, C. Léandri, S. Vizzini, C. Quaresima, P. Perfetti, B. Olivieri, H. Oughaddou, B. Aufray, and G. Le Lay, Nano Lett. 8, 2299 (2008).

${ }^{13}$ B. Aufray, A. Kara, S. Vizzini, H. Oughaddou, C. Léandri, B. Ealet, and G. Le Lay, Appl. Phys. Lett. 96, 183102 (2010).

${ }^{14}$ A. Kara, S. Vizzini, C. Léandri, B. Ealet, H. Oughaddou, B. Aufray, and G. Le Lay, J. Phys.: Condens. Matter 22, 045004 (2010).

${ }^{15}$ P. De Padova, C. Quaresima, C. Ottaviani, P. M. Sheverdyaeva, P. Moras, C. Carbone, D. Topwal, B. Olivieri, A. Kara, H. Oughaddou, B. Aufray, and G. Le Lay, Appl. Phys. Lett. 96, 261905 (2010).

${ }^{16}$ E. Loginova, S. Nie, K. Thürmer, N. C. Bartelt, and K. F. McCarty, Phys. Rev. B 80, 085430 (2009).

${ }^{17}$ P. Sutter, J. T. Sadowski, and E. Sutter, Phys. Rev. B 80, 245411 (2009) 